\title{
Concentración de lactato en voleibolistas profesionales universitarios $^{1}$
}

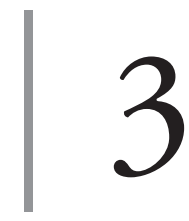

\author{
https://doi.org/10.21830/9789585284814.03
}

Carlos Enrique Cabrera Arismendy², Diana María García-Cardona ${ }^{3}$,
Oscar Eduardo Sánchez Muñoz

\section{Resumen}

Objetivo: determinar la concentración de lactato de los estudiantes deportistas pertenecientes a la selección de voleibol de la Universidad del Quindío. Metodología: se utilizó un enfoque cuantitativo y descriptivo. Se tomaron tres muestras de sangre para determinar la concentración de lactato: la primera, inmediatamente antes de comenzar el calentamiento para la prueba; la segunda, inmediatamente terminada la prueba; y la tercera, diez minutos después de terminada la prueba (Test Legger-Lambert). Además, se determinaron las variables edad, talla, masa corporal, IMC, consumo máximo de oxígeno y frecuencia cardiaca. Resultados: se pudo establecer el comportamiento del lactato; se presentó normalidad en ambos sexos, a excepción de algunos datos. Con referencia al $\mathrm{VO}_{2 \max }$, se encontró mayor consumo en los hombres que en las mujeres; sin embargo, en promedio, ambos grupos se encontraron dentro de la normalidad. La frecuencia cardiaca tuvo un comportamiento progresivo, de acuerdo con el esfuerzo realizado, pues fue más alta al finalizar la prueba. Conclusión: al relacionar el comportamiento de la frecuencia cardiaca, $\mathrm{VO}_{2 \max }$ y el lactato se evidenció, en algunos casos, la presencia de deportistas

1 Este texto hace parte de los procesos misionales que la Universidad del Quindío contempla como parte fundamental de la responsabilidad académica de los docentes. Ha sido posible a través de los apoyos económicos para realizar investigación por medio de las facultades, los grupos y las líneas de investigación reconocidas por Colciencias y la institución. Es el producto de los permanentes desarrollos realizados en la Facultad de Educación por el programa de Licenciatura en Educación Física y Deportes, en la línea de investigación "Entrenamiento deportivo".

2 Magíster en Educación y desarrollo humano, docente programa de Licenciatura en Educación Física y Deportes de la Universidad del Quindío. Contacto: cecabrera@uniquindio.edu.co. Orcid: https:// orcid.org/0000-0001-5581-0543

3 Magíster en Ciencias Biomédicas, docente programa de Licenciatura en Educación Física y Deportes de la Universidad del Quindío. Contacto: dmgarcia@uniquindio.edu.co. Orcid: https://orcid. org/0000-0001-6026-9093

4 Magíster en Educación, docente programa de Licenciatura en Educación Física y Deportes de la Universidad del Quindío. Contacto: oesanchez@uniquindio.edu.co. Orcid: https://orcid.org/0000-0003$2438-3360$ 
con baja preparación física, lo que implica niveles bajos de consumo máximo de oxígeno, altos promedios de frecuencia cardiaca y poca concentración de lactato; en otros casos, se evidenció que la concentración fue alta, pero su desempeño mostró buena potencia aeróbica al marcar niveles altos en el test.

Palabras clave: consumo máximo de oxígeno; estudiantes universitarios; frecuencia cardiaca; lactato.

\section{Introducción}

El voleibol es un deporte de conjunto que requiere de una preparación física específica, implicando de esta manera el reconocimiento por parte de los entrenadores de las dinámicas del juego y su desenvolvimiento en términos de gasto energético, para un buen desempeño en la competencia. Tal y como lo argumenta Ivoilov (1986):

El vóleibol se considera como un deporte con grandes gastos de energía, por lo cual se encuentra clasificado como un deporte de potencia submáxima que se caracteriza por la alternancia de fases activas de trabajo con pausas breves de descanso relativamente pasivas, las múltiples repeticiones de exigencias de carácter alternado y con breves pausas de descanso hacen hincapié del rendimiento aeróbico-anaeróbico. En resumen, el vóleibol es considerado como un deporte de equipo que requiere una considerable capacidad de resistencia, y que si bien algunas consideraciones para aumentar el desempeño de los atletas, será un entrenamiento intenso aeróbico a nivel del mar, lo cual incrementaría los niveles de $\mathrm{VO}_{\text {2máx }}$ del deportista con lo cual les permitirá competir en determinadas altitudes. (p. 157)

En este proyecto se hizo énfasis en una necesidad recurrente de los deportistas: contar con las herramientas necesarias para determinar de manera clara aspectos condicionantes a nivel fisiológico que son importantes para mejorar y alcanzar un óptimo rendimiento deportivo, a través de la planificación de sus ciclos de competencia y entrenamiento diarios.

Dentro de dichos aspectos fisiológicos se encuentra la concentración de lactato, porque: "el ácido láctico es un producto que se genera en el músculo ante esfuerzos intensos como resultado de procesos metabólicos para generar energía en un corto tiempo" (López, 2002, p. 148). De hecho, "como último recurso para los músculos exhaustos que han sido sometidos a ejercicio intenso 
debido al consumo rápido de oxígeno y a la falta de metabolismo aeróbico de la glucosa (Atkins, 2007, p. 240). Razón por la cual, algunos autores mencionan que el límite anaeróbico de ácido láctico se sitúa en $4 \mathrm{mmol}$ por litro de sangre. Sin embargo, este límite puede ser muy variable según la intensidad del esfuerzo en cada sujeto, por lo que se considera difícil establecer una base para la concentración de ácido láctico (Ahonen et al., 2001). Otros autores, como Urdampilleta, Martínez y López (2013), sostienen que valores de ácido láctico que se encuentren por encima de los $4 \mathrm{mmol} / \mathrm{L}$ son indicadores del aumento de la intensidad del entrenamiento.

Ahora bien, la concentración de lactato en sangre ha sido utilizada para valorar el rendimiento específico de los deportistas, ya que el ácido láctico es un factor que tiene un importante papel como indicador de la intensidad en la mayoría de los deportes, siendo también un parámetro razonable para la estimación de la intensidad de la carga de trabajo durante el entrenamiento, lo que ayuda a establecer, en forma individual y objetiva, la intensidad del ejercicio a la que se quiere trabajar. Además, la presente investigación tuvo como referencia la necesidad de hacer que los procesos deportivos institucionales comiencen a tener mayor visibilidad científica, con el fin de permitir a los entrenadores un mayor conocimiento tanto de los deportistas como de los procesos que se pueden llevar a cabo a partir de dicho conocimiento. De tal forma que el objetivo general de la investigación fue determinar la concentración de lactato de los estudiantes deportistas pertenecientes a la selección de voleibol de la Universidad del Quindío, resultados fueron relacionados con variables como el consumo máximo de oxígeno $\left(\mathrm{VO}_{2 \max }\right)$ y la frecuencia cardiaca.

\section{Metodología}

El tipo de estudio fue descriptivo y exploratorio de corte transversal. Para tal efecto, se trabajó con veinticuatro (24) deportistas, lo que corresponde al total de los estudiantes pertenecientes a las selecciones femenina (10) y masculina (14) de voleibol de la Universidad del Quindío.

Cabe aclarar que se excluyeron de la investigación los estudiantes deportistas que no firmaron el consentimiento informado, que presentaban lesión o que se encontraban en recuperación de lesión al momento de realizar las evaluaciones. 
Por lo tanto, las personas que participaron lo hicieron de manera voluntaria; de modo que se buscó proporcionar una adecuada información y comprensión por medio del consentimiento informado. Todas las inquietudes que se generaron fueron resueltas por los investigadores, con miras a garantizar una adecuada comunicación y comprensión por parte de los participantes, para que su consentimiento voluntario fuera real y pudieran, en el momento que lo consideraran, abandonar su participación en el proyecto. Frente a estas consideraciones éticas, el presente estudio obtuvo el aval del Comité de ética y bioética de investigación de la Universidad del Quindío, en el acta 24 del 14 de agosto de 2015.

La información se recogió por los investigadores a partir de la fuente primaria, por medio de un instrumento diseñado para tal fin con todas las variables del estudio.

Con respecto a las variables antropométricas, las medidas se realizaron partiendo de la posición antropométrica de referencia, se midió la masa (la cual fue tomada en la mañana previamente al desayuno, después de haber miccionado y con el mínimo de ropa posible), la talla y el Índice de Masa Corporal (IMC). La frecuencia cardiaca se tomó con un pulsómetro (POLAR FT1).

Con respecto a la concentración de lactato se tomaron 3 muestras de la siguiente forma: la primera (basal), inmediatamente antes de comenzar el calentamiento para la prueba; la segunda (postest), inmediatamente terminada la prueba, según las recomendaciones del test Leger-Lambert (1982) y, la tercera (recuperación) diez minutos después de terminada la prueba.

Cada extracción de sangre se realizó por punción en el dedo, bajo condiciones de asepsia y antisepsia (con una lanceta de uso único esterilizada). La concentración de lactato se determinó con tiras reactivas en el equipo Lactate Scout, siguiendo las instrucciones del fabricante.

Se procesó la información y se hizo un análisis descriptivo de cada una de las variables, los resultados se presentan con el promedio \pm la desviación estándar y los límites máximos y mínimos. Se realizó una Anova de medidas repetidas, igualmente se utilizó la "T" de Student, se consideró la significancia estadística de un P-valor $\leq 0,05$. El procesamiento de los datos obtenidos se realizó en el software spss versión 10.0. 


\section{Resultados}

Los voleibolistas presentaron en promedio una edad de 20,8 años, la media por sexo fue de 21,3 años para mujeres y 20,3 años para los hombres. En la Tabla 1 se presentan las variables antropométricas por sexo en las cuales se refleja de manera general el estado corporal de los estudiantes deportistas participantes del estudio.

Tabla 1. Variables antropométricas

\begin{tabular}{lccc}
\hline Variables & \multicolumn{1}{c}{$\begin{array}{c}\text { Población total } \\
\mathbf{n = 2 4}\end{array}$} & \multicolumn{1}{c}{$\begin{array}{c}\text { Mujeres } \\
\mathbf{n}=\mathbf{1 0}\end{array}$} & \multicolumn{1}{c}{$\begin{array}{c}\text { Hombres } \\
\mathbf{n}=\mathbf{1 4}\end{array}$} \\
\hline Talla $(\mathrm{cm})$ & $172 \pm 10,8(154-191)$ & $162 \pm 5,7(154-172)$ & $179 \pm 7,2(164-191)$ \\
Masa $(\mathrm{Kg})$ & $67,6 \pm 8,3(54-78,3)$ & $60,8 \pm 5,1(54-68)$ & $74,5 \pm 6,5(61,6-78,3)$ \\
$\begin{array}{l}\mathrm{IMC} \\
\left(\mathrm{kg} / \mathrm{m}^{2}\right)\end{array}$ & $22,83 \pm 2,2(20,44-28,88)$ & $23,21 \pm 7,1(20,86-28,88)$ & $22,55 \pm 8,8(20,44-26,87)$ \\
\hline
\end{tabular}

Fuente: elaboración propia.

Con relación al índice de masa muscular, se encontró que, en promedio, los deportistas se encuentran en el rango normal, según la OMS (2016); no obstante, existen dos casos de sobrepeso (un hombre y una mujer).

A continuación, se presenta la frecuencia cardiaca, el consumo máximo de oxígeno y la concentración de lactato en los deportistas.

Tabla 2. Frecuencia cardiaca, $\mathrm{VO}_{2 \max }$ y concentración de lactato

\begin{tabular}{lccc}
\hline Variables & $\begin{array}{c}\text { Población total } \\
\mathbf{n = 2 4}\end{array}$ & $\begin{array}{c}\text { Mujeres } \\
\mathbf{n = 1 0}\end{array}$ & $\begin{array}{c}\text { Hombres } \\
\mathbf{n}=\mathbf{1 4}\end{array}$ \\
\hline FC1 & $95 \pm 15,9(75-128)$ & $97 \pm 15,9(82-128)$ & $93 \pm 16,2(75-121)$ \\
FC2 & $193 \pm 9,8(170-206)$ & $190 \pm 6,1(181-202)$ & $196 \pm 11,4(170-206)$ \\
\hline
\end{tabular}




\begin{tabular}{lccc}
\hline Variables & \multicolumn{1}{c}{$\begin{array}{c}\text { Población total } \\
\mathbf{n = 2 4}\end{array}$} & $\begin{array}{c}\text { Mujeres } \\
\mathbf{n = 1 0}\end{array}$ & $\begin{array}{c}\text { Hombres } \\
\mathbf{n}=\mathbf{1 4}\end{array}$ \\
\hline FC3 & $119 \pm 12,1(95-150)$ & $118 \pm 6,7(111-135)$ & $119 \pm 12,1(95-150)$ \\
$\begin{array}{l}\text { Lact 1 } \\
(\mathrm{mmol} / \mathrm{l})\end{array}$ & $1,49 \pm 0,5(0,6-2,8)$ & $1,48 \pm 6,6(0,6-2,8)$ & $1,5 \pm 2,5(0,8-2,4)$ \\
$\begin{array}{l}\text { Lact 2 } \\
(\mathrm{mmol} / \mathrm{l})\end{array}$ & $11,03 \pm 3,5(4,0-16,4)$ & $9,36 \pm 3,5(5,6-16,4)$ & $11,7 \pm 3,1(4,0-15,1)$ \\
$\begin{array}{l}\text { Lact 3 } \\
(\mathrm{mmol} / \mathrm{l})\end{array}$ & $8,4 \pm 3,3(4,6-13)$ & $6,8 \pm 3,3(4,6-12,7)$ & $9,23 \pm 3,2(6,9-13)$ \\
$\begin{array}{l}\mathrm{VO} 2 \mathrm{MAX} \\
(\mathrm{ml} / \mathrm{kg} / \mathrm{min}\end{array}$ & $52,2 \pm 5,9(42,07-62,58)$ & $48,2 \pm 5,4(42,07-59,6)$ & $55,1 \pm 4,6(45-62,58)$ \\
\hline
\end{tabular}

Fuente: elaboración propia (2017).

FC1: frecuencia cardiaca basal. FC2: frecuencia cardiaca finalizada la prueba. FC3: frecuencia cardiaca tras reposos de 10 minutos. Lactato 1: concentración de lactato basal. Lactato 2: concentración de lactato finalizada la prueba. Lactato 3: concentración de lactato tras reposos de 10 minutos. $\mathrm{VO}_{2 \max }$ : consumo máximo de oxígeno.

En un sentido amplio, el grupo de deportistas, hombres y mujeres, tuvo una frecuencia cardiaca basal menor a 100 pulsaciones por minuto. Al respecto, López (2009) señal que "el pulso es el principal indicador de esfuerzo cardiovascular, por lo tanto, esto lo convierte en un determinante de la intensidad del ejercicio" (p. 150). En este contexto, el promedio general obtenido en el FC2, 193 ppm, indica un alto esfuerzo realizado en la prueba. Por sexo se observó que el promedio en mujeres y hombres fue de 190 y 196 ppm respectivamente, lo que indica promedios parecidos en su comportamiento. En el caso del tercer momento de toma (FC3), se considera que los deportistas tuvieron un comportamiento normal al estar plenamente recuperados.

Asimismo, se analizó el consumo máximo de oxígeno $\left(\mathrm{VO}_{2 \max }\right)$, el cual es uno de los mejores indicadores de la condición cardiorrespiratoria; es decir, uno de los parámetros más importantes de los niveles de acondicionamiento físico de un atleta. Por tal motivo, el $\mathrm{VO}_{2 \max }$ puede ser visto en su compor- 
tamiento con relación a la frecuencia cardiaca, con el fin de determinar los niveles de esfuerzo durante la prueba (American College of Sport Medicine, 2006, p. 300). Por su parte, Zintl (citado por Ramos, Melo \& Alzate, 2007) sostiene, que "el consumo máximo de oxígeno $\left(\mathrm{VO}_{2 \max }\right)$ es una medida para el aporte de oxígeno (respiración), el transporte de oxígeno (sistema cardiovascular) y la utilización de oxígeno (célula muscular) en un organismo esforzado al máximo" (p. 146).

En lo que tiene que ver con la concentración de lactato, en función del momento de la toma de la muestra, los datos corroboran que existen diferencias entre las medias de la concentración de lactato (Figura 1) entre la muestra basal, postest y tras 10 minutos de reposo $(\mathrm{p}<0.05)$, a nivel general y según el sexo. Sin embargo, no hubo diferencias estadísticamente significativas al comparar cada uno de los momentos (basal P-valor $=0,9542$, postest P-valor $=0,3677$, reposo $\mathrm{P}$-valor $=0,0973$ ) entre hombres y mujeres.

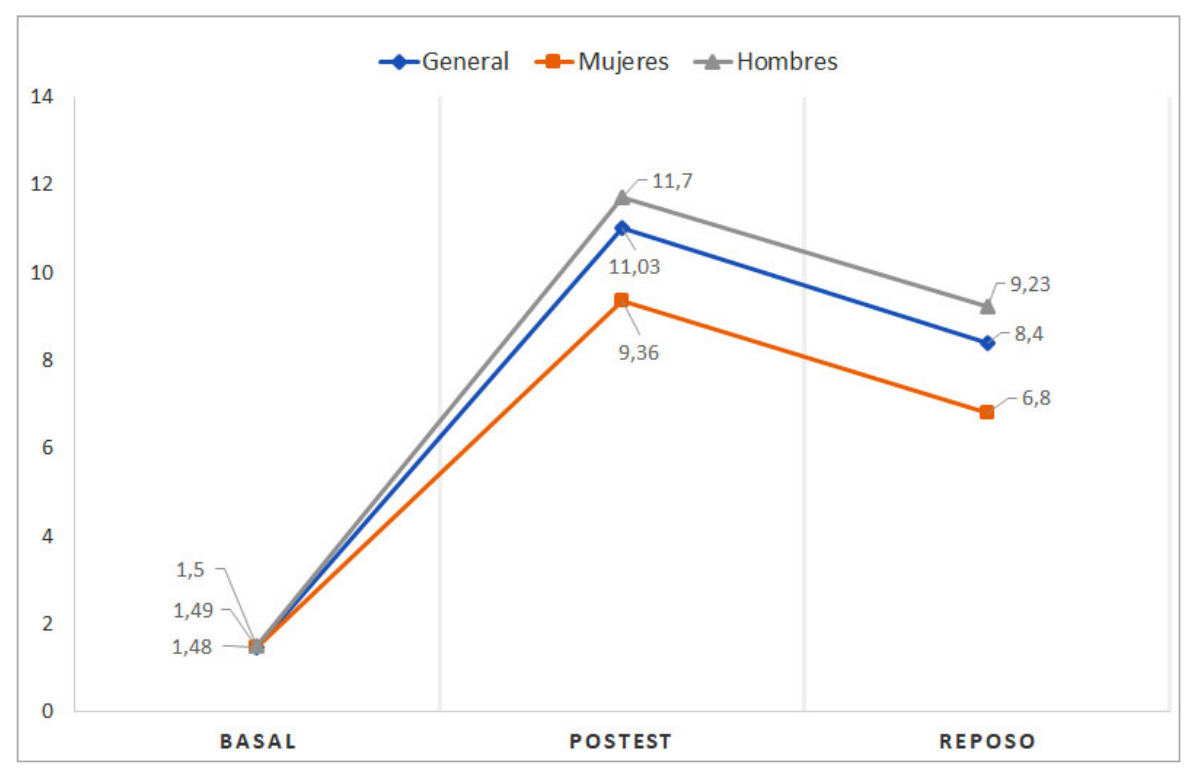

Figura 1. Momento y promedio de la concentración de lactato por sexo. Fuente: elaboración propia (2017).

A continuación, se muestran las tablas 3 y 4 , las cuales muestran los resultados de la segunda muestra en hombres y mujeres. 
Tabla 3. Variables posesfuerzo de hombres

\begin{tabular}{|c|c|c|c|c|c|}
\hline $\begin{array}{c}\text { Rango de concentración } \\
\text { de lactato }\end{array}$ & $\mathrm{n}=14$ & $\begin{array}{l}\text { Nivel } \\
\text { (Fr ab) }\end{array}$ & $\mathrm{VO}_{2 \max }$ & Lactato 2 & FC2 \\
\hline $4-5,99 \mathrm{mmol} / \mathrm{l}$ & 1 & 6 & & & $\begin{array}{c}196 \pm 11 \\
(170-206)\end{array}$ \\
\hline $\begin{array}{l}\text { Mayor o igual } \\
\text { a } 8 \mathrm{mmol} / \mathrm{l}\end{array}$ & 13 & $\begin{array}{c}8=3 \\
9=2 \\
10=5 \\
11=2 \\
12=1\end{array}$ & $\begin{array}{c}55,5 \pm 22 \\
(45-62,58)\end{array}$ & $\begin{array}{l}11,47 \pm 1,9 \\
(10,6-15)\end{array}$ & \\
\hline
\end{tabular}

Fr ab: frecuencia absoluta. FC2: frecuencia cardiaca finalizada la prueba. Lactato 2: concentración de lactato finalizada la prueba. $\mathrm{VO}_{2 \max }$ consumo máximo de oxígeno.

Fuente: elaboración propia (2017).

Tabla 4. Variables posesfuerzo de mujeres

\begin{tabular}{|c|c|c|c|c|c|}
\hline $\begin{array}{c}\text { Rango de concentración } \\
\text { de lactato }\end{array}$ & $n=10$ & $\begin{array}{l}\text { Nivel } \\
\text { (Fr ab) }\end{array}$ & $\mathrm{VO}_{2 \max }$ & Lact 2, & FC2, \\
\hline $6-7,99 \mathrm{mmol} / \mathrm{l}$ & 2 & $\begin{array}{l}5=1 \\
6=1\end{array}$ & $(42,07-45)^{*}$ & $(5,6-7,9)^{*}$ & $(193-192)^{*}$ \\
\hline $\begin{array}{l}\text { Mayor o igual } \\
\text { a } 8 \mathrm{mmol} / \mathrm{l}\end{array}$ & 8 & $\begin{array}{c}5=1 \\
6=1 \\
7=3 \\
8=1 \\
9=1 \\
11=1\end{array}$ & $\begin{array}{c}48,76 \pm 5,5 \\
(42,07- \\
59,75)\end{array}$ & $\begin{array}{c}9,36 \pm 2 \\
(8-15)\end{array}$ & $\begin{array}{c}188 \pm 5,4 \\
(181-198)\end{array}$ \\
\hline
\end{tabular}

Fr ab: frecuencia absoluta. FC2: frecuencia cardiaca finalizada la prueba. Lactato 2: concentración de lactato finalizada la prueba. $\mathrm{VO}_{2 \max }$ c consumo máximo de oxígeno.

Fuente: elaboración propia (2017).

En el análisis individual un deportista alcanzó etapa $12, \mathrm{VO}_{2 \max }$ de 62,58 $\mathrm{ml} / \mathrm{kg} / \mathrm{min}$, concentración de lactato $14,4 \mathrm{mmol} / \mathrm{l}$, y frecuencia cardiaca inmediatamente finalizada la prueba (FC2) de 205 ppm; estos datos describen a un deportista con buen desarrollo de la potencia aeróbica máxima, por lo tanto, con capacidad de ejecutar esfuerzos en la zona aeróbica-anaeróbica, respondiendo bien a esfuerzos anaeróbicos lácticos y con buena tolerancia y aprovechamiento del ácido láctico. 
En el caso de las mujeres, el comportamiento del lactato (lactato 2), la frecuencia cardiaca (FC2) y el $\mathrm{VO}_{2 \max }$ a través del desarrollo de la prueba (etapa alcanzada), mostrados en la Tabla 4, presentan dos grupos en el rango de concentración de lactato: el primero, conformado por dos mujeres (zona anaeróbica durmiente, correspondiente con cifras de lactato de 6 a $8 \mathrm{mmol} / \mathrm{l}$, en esta zona no hay estímulo para el desarrollo de la capacidad anaeróbica, pero sí existe un aumento de las posibilidades aeróbicas, y el segundo, conformado por ocho mujeres (zona anaeróbica estimulante). Según esto, se encontró que el grupo dos se encuentra en mejor condición física que el grupo uno, lo que llevó a alcanzar mejores etapas en la prueba y, por lo tanto, mejores niveles de $\mathrm{VO}_{2 \max }(48,76 \mathrm{ml} / \mathrm{kg} / \mathrm{min})$, que según la referencia de Astrand y Rodahl (1986) es muy buena; además, el esfuerzo realizado por las deportistas fue alto, esto, de acuerdo con la frecuencia cardiaca promedio alcanzada (188 ppm), lo que permite decir que estas mujeres lograron llegar a esfuerzos de potencia aeróbica máxima y potencia anaeróbica submáxima, y hasta casi máxima, lo que pudo haber llevado a mayor concentración de lactato, a partir de su buena condición atlética (López, 2006).

\section{Discusión}

En las variables antropométricas se encontró, con respecto a la talla, un promedio general de $172 \mathrm{~cm}$. Para el caso de las mujeres fue de $162 \mathrm{~cm}$ y de los hombres de $179 \mathrm{~cm}$. En el ámbito internacional se encuentran estudios que definen parámetros similares a los promedios encontrados en este estudio, donde se aprecia promedios en deportistas con una talla de $181 \mathrm{~cm}$; estos datos, tomados de universitarios espańoles y juveniles brasileńos, muestran diferencias de $9 \mathrm{~cm}$, lo que evidencia una talla más baja en nuestra región (Cabral et al., 2011). Por otra parte, cabe destacar que estos datos corresponden a la potencia suramericana y mundial en voleibol, además, que España ha demostrado avances en los procesos formativos de sus deportistas.

Otro referente que bien vale la pena mencionar es el realizado por la Universidad de Ciencias Informáticas de La Habana, Cuba, en el cual existe similitud en lo que tiene que ver con los sujetos deportistas universitarios con 
una edad promedio de 23 años, los cuales también participan de certámenes regionales y nacionales como sucede con nuestros estudiantes deportistas. Para este caso el promedio en la talla es de $188 \mathrm{~cm}$ (Pérez, Portela, Cantero \& Rodríguez, 2014).

Ahora bien, en los deportistas uniquindianos existe una diferencia de 9,6 $\mathrm{cm}$ por debajo con respecto a los contextos mencionados anteriormente. Esto permite manifestar que, aunque estos valores no superan $l o s \quad 10 \mathrm{~cm}$ de diferencia, la variable antropométrica, probablemente, puede ser determinante en el desempeño y rendimiento en la práctica de esta disciplina deportiva. Es más, según Norton y Olds (1996), en el voleibol la altura de la malla se encuentra a 2,43 metros, por lo tanto, aquellos jugadores más altos tienen que realizar esfuerzos en salto relativamente menores para poder superar la altura de la red. Los bloqueadores y rematadores ocupan alrededor de 7 a 15 minutos en un partido, realizando esfuerzos en saltos, por lo que la consecución de repetidos saltos explosivos es, junto con la talla, aspectos claves en el éxito (p. 398).

De otra parte, existe gran interés por definir las diferencias estructurales en los sujetos de diversas disciplinas deportivas en el ámbito universitario; para el caso del voleibol, las variables antropométricas son parámetros fundamentales y decisivos en el rendimiento. Así mismo, diversos estudios como el de García-Cardona et al., (2017) han demostrado que la talla en los deportistas es una condición estructural favorable para la práctica del voleibol y, por ende, un factor determinante en el desempeño.

El voleibol, al ser un deporte que durante el juego tiene picos de esfuerzo y donde la potencia muscular resulta fundamental para la ejecución de diferentes movimientos técnicos, también requiere, a lo largo del partido, una buena capacidad aeróbica que permita al deportista llegar hasta los últimos momentos del juego. Por lo anterior, desde el punto de vista funcional, es primordial el desarrollo de la velocidad-fuerza y una óptima base aeróbica que permita la recuperación adecuada del atleta, a esto hay que agregar que la evaluación del comportamiento en varios aspectos fisiológicos resulta determinante para identificar la capacidad del deportista y valorar su condición física. En promedio, el grupo de deportistas de ambos sexos manejaron una frecuencia cardiaca basal menor a 100 pulsaciones por minuto. Según lo 
propuesto por López: "el pulso es el principal indicador de esfuerzo cardiovascular, por lo tanto, esto lo convierte en un determinante de la intensidad del ejercicio" (2009, p. 150). A este respecto, el promedio general obtenido en el FC2 fue de 193 ppm, lo que indica que el esfuerzo realizado en la prueba fue alto; el promedio en mujeres y hombres fue de 190 y 196 ppm respectivamente, lo que indica un comportamiento similar en ambos sexos. En el caso del tercer momento de toma (FC3), se pudo concluir que los deportistas tuvieron un comportamiento normal al estar plenamente recuperados.

De la misma forma, se analizó el consumo máximo de oxígeno $\left(\mathrm{VO}_{2 \max }\right)$, el cual es uno de los mejores indicadores de la condición cardiorrespiratoria, es decir, uno de los parámetros más importantes de los niveles de acondicionamiento físico de un atleta. $\mathrm{El} \mathrm{VO}_{2 \max }$ puede ser visto en su comportamiento con relación a la frecuencia cardiaca, con el fin de determinar los niveles de esfuerzo durante la prueba. Según Zintl (citado por Ramos, Melo \& Alzate, 2007), "el consumo máximo de oxígeno $\left(\mathrm{VO}_{2 \max }\right)$ es una medida para el aporte de oxígeno (respiración), su transporte de oxígeno (sistema cardiovascular) y la utilización de oxígeno (célula muscular) en un organismo esforzado al máximo" (p. 146). En el $\mathrm{VO}_{2 \max }$ de la muestra evaluada se puede identificar un promedio mayor de consumo por parte de los hombres $(55,1 \mathrm{ml} / \mathrm{kg} / \mathrm{min})$ con respecto a las mujeres $(48,2 \mathrm{ml} / \mathrm{kg} / \mathrm{min})$. Desde el punto de vista comparativo, existen estudios cercanos como el de Zanella et al., (2015) en el que la valoración de grupos de voleibolistas masculinos brasileros por posición de juego, con promedio de edad de 23 años, mostraron valores similares a los encontrados en este estudio. Además, el parámetro fue excelente, según la clasificación de Astrand \& Rodahl (1986), para esta variable. Por otro lado, se encontró que en la evaluación realizada a 170 mujeres argentinas voleibolistas de diferentes categorías (19), todas tenían un $\mathrm{VO}_{2 \max }$ similar $(-42,3 \mathrm{ml} / \mathrm{kg} /$ min), valores que están por debajo de los encontrados en nuestra población.

Con base en las variables de frecuencia cardiaca y $\mathrm{VO}_{2 \max }$, se puede inferir que el comportamiento de los deportistas uniquindianos varía según la condición física y el sexo, pues la mayoría de los hombres tuvieron mayor capacidad de consumo, al parecer, debido a sus condiciones fisiológicas. También es importante reconocer que al ser una disciplina deportiva donde los esfuerzos 
se alternan durante la competencia, no se especifica en la base aeróbica, por lo cual su $\mathrm{VO}_{2 \max }$ no alcanza rangos tan importantes como los deportistas de pruebas de fondo (maratonistas, ciclistas, triatletas, entre otros). Con base en lo anterior, se podría establecer que estos deportistas muestran niveles de preparación física adecuados y acordes con las sesiones de entrenamiento que aplican semanalmente (5 días).

Con relación a la concentración de lactato en función del momento de la toma de la muestra, los datos corroboran que existen diferencias entre las medias de la concentración de lactato entre la muestra basal, postest y tras diez minutos de reposo $(\mathrm{p}<0.05)$, a nivel general y según el sexo. Sin embargo, no hubo diferencias estadísticamente significativas al comparar cada uno de los momentos entre hombres y mujeres. Tal y como lo plantean Earle y Baechle (2007),

en cuanto a la producción, unos mayores niveles de ácido láctico significan una mayor intervención de la glucolisis rápida, lo cual conlleva a una mayor potencia de producción de ATP y por tanto, una mayor velocidad de carrera. No obstante, un exceso de producción de ácido láctico, sin su correcta reutilización, significaría la aparición de fatiga muscular y obligaría a descender el rendimiento drásticamente. (p. 700)

Según López (2006), valores de lactato iguales o superiores a $4 \mathrm{mmol} / \mathrm{l}$ se corresponden con el momento previo al rompimiento del equilibrio entre la producción y la remoción del lactato en sangre. Según el tipo de deporte que ha sido evaluado, se puede determinar que los esfuerzos combinados, aeróbico y anaeróbicos, permiten la organización de un entrenamiento que apoye el mejoramiento de las dos vías, en especial la potencia aeróbica máxima y potencia anaeróbica máxima y submáxima, pues, como se anotó anteriormente, el voleibol es un deporte variable, en el que se alternan fases de trabajo aeróbico y anaeróbico. El tiempo anaeróbico se manifiesta fundamentalmente en los saltos y la cualidad aeróbica está implícita en los momentos que no se están realizando acciones de ataque o defensa producidas a lo largo del juego.

Con base en el comportamiento del lactato, en la evaluación realizada se encontró que en ninguno de los grupos de la muestra 1 (Lactato 1, basal) se presentó una concentración por fuera de la normalidad. Esto, con respecto a 
los presupuestos de López (2006), indica que los sujetos se encontraban en la zona aeróbica compensadora, lo cual indica una relajación antes de la aplicación del test Leger-Lambert, lo que corresponde a valores de lactato ubicados en un rango entre 0 y $2 \mathrm{mmol} / \mathrm{l}$. En esta zona no se busca un desarrollo de la capacidad de resistencia general, pues su frecuencia de trabajo es muy baja, por lo tanto, no hay un incremento de la concentración (como sucede, por ejemplo, en el calentamiento y el enfriamiento).

Con respecto a la segunda muestra (FC2 y Lact 2), se encontró que los hombres, en cuanto a la concentración de lactato, se ubicaron básicamente en dos rangos o zonas de entrenamiento: zona aeróbica no estimulante y zona anaeróbica estimulante. De forma general, es un comportamiento que puede ser analizado como normal en el $\mathrm{VO}_{2 \max }$, siempre y cuando se hable de personas normales, pero para el caso de deportistas debería ser mejor, aún más si se toma en cuenta que la concentración de lactato alcanzada se mueve en una zona aeróbica no estimulante y se especifica en la capacidad aeróbica, lo que nos hace pensar que el test lo llevó a una fatiga rápida, con una FC alta, a pesar de no haber llegado a una etapa donde se exprese alto esfuerzo, de lo que se podría inferir que se trata de un deportista sin condiciones para trabajar con mayor intensidad. López (2006) agrega que la zona aeróbica no estimulante se utiliza dentro del proceso de entrenamiento en deportes de baja intensidad y alto volumen, así la energética utilizada es la de los carbohidratos y los lípidos, que se corresponde con los valores de lactato que oscilan entre 2 a $4 \mathrm{mmol} / \mathrm{l}$.

En el caso de la segunda zona utilizada se encuentran los 13 deportistas restantes, quienes lograron alcanzar etapas entre 8 y 12 en el test propuesto, con un $\mathrm{VO}_{2 \max }$ promedio de $55.81 \mathrm{ml} / \mathrm{kg} / \mathrm{min}$, FC de 196 ppm y una concentración de lactato de $11,7 \mathrm{mmol} / \mathrm{l}$. Al relacionar las tres variables se puede advertir que se trata de un grupo de deportistas con un buen nivel de $\mathrm{VO}_{2 \max }$, puesto que alcanzaron etapas en la prueba por encima de 8 , lo que representa una capacidad aeróbica adecuada; además, la frecuencia cardiaca muestra un nivel de intensidad alta generada durante la prueba, que demuestra, de acuerdo con los rangos establecidos (170-206 ppm) que los estudiantes se esforzaron al límite de su capacidad de resistencia. Con relación a la variable lactato, los rangos que se establecieron $(10,6-15 \mathrm{mmol} / \mathrm{l})$ demuestran concentraciones 
de lactato relativamente altas con relación al esfuerzo aplicado en la prueba. Según López (2006), la zona anaeróbica estimulante es un rango de entrenamiento en el cual se entrenan las capacidades anaeróbicas que se ubica entre 8 y $12 \mathrm{mmol} / \mathrm{l}$ de lactato.

En promedio, los valores encontrados en la concentración de lactato (Lac 2) en hombres está por debajo de los valores medios de lactato reportados por Engel, Härtel, Wagner, Strahler, Bös y Sperlich (2014) que indican 16,3 $\mathrm{mmol} / \mathrm{l}$ en adultos durante un ejercicio de alta intensidad en cicloergómetro.

Individualmente se pudo apreciar a un deportista que alcanzó etapa $12, \mathrm{VO}_{2 \max }$ de $62,58 \mathrm{ml} / \mathrm{kg} / \mathrm{min}$, concentración de lactato $14,4 \mathrm{mmol} / \mathrm{l} \mathrm{y}$ frecuencia cardiaca, inmediatamente finalizada la prueba (FC2), de 205 ppm; estos datos describen a un deportista con buen desarrollo de la potencia aeróbica máxima, por lo tanto, con capacidad de ejecutar esfuerzos en la zona aeróbica-anaeróbica, que responde bien a esfuerzos anaeróbicos lácticos, que posee buena tolerancia y aprovecha de manera eficiente el ácido láctico. De acuerdo a lo planteado por Alonso et al., (2012),

en relación con la eliminación o aclaramiento del ácido láctico, es imprescindible la capacidad del deportista de utilizar dicho producto como sustrato energético, por un lado, evitar la acumulación de metabolitos que produzcan fatiga y, por otro, aportar unas moléculas de ATP extras que ayuden a mantener la contracción muscular. (p. 95)

Esta apreciación, en consonancia con la preparación del deportista, suele dar pie a afirmar que una buena reutilización del lactato depende, en gran medida, del trabajo que se haga en la zona de tolerancia, lo que implica adaptar sus condiciones fisiológicas para generar energía a partir del ácido láctico, esto indica que sin una buena preparación física, especialmente en la zona adecuada, será difícil hacer que el deportista pueda alcanzar buena tolerancia al lactato y se pueda desempeñar satisfactoriamente en su presencia. Según Alonso et al. (2012):

dicha reutilización se produce de diversas maneras. Así, mediante el ciclo de Cori, el lactato pasa a la sangre y llega al hígado, donde es convertido en glucosa. Por otro lado, mediante la glucolisis lenta o aeróbica, el ácido láctico se convierte en piruvato, el cual es utilizado como sustrato en la oxidación de 
la glucosa en el ciclo de Krebs [...], de este modo la capacidad del deportista de utilizar este tipo de medios, optimizada gracias al entrenamiento, es imprescindible para evitar la acumulación de ácido láctico y lograr así mantener la intensidad del esfuerzo por más tiempo. (p. 95)

En el caso de las mujeres, el comportamiento del lactato (lactato 2), la frecuencia cardiaca (FC2) y el $\mathrm{VO}_{2 \max }$ a través del desarrollo de la prueba (etapa alcanzada), que se muestran en la Tabla 4, presentan dos grupos en el rango de concentración de lactato: el primero conformado por dos mujeres (zona anaeróbica durmiente, correspondiente con cifras de lactato de 6 a $8 \mathrm{mmol} / \mathrm{l}$, en esta zona no hay estímulo para el desarrollo de la capacidad anaeróbica pero sí existe un aumento de las posibilidades aeróbicas y el segundo por ocho (zona anaeróbica estimulante). Según esto, se encontró que el grupo dos se encuentra en mejor condición física que el grupo uno, lo que le permitió alcanzar mejores etapas en la prueba y, por tanto, mejores niveles de $\mathrm{VO}_{2 \max }(48,76 \mathrm{ml} / \mathrm{kg} /$ min) que, según la referencia de Astrand y Rodahl (1986), es muy buena. Además, se puede demostrar que el esfuerzo realizado por las deportistas fue alto, de acuerdo con la frecuencia cardiaca promedio alcanzada (188 ppm), lo que permite analizar que estas mujeres lograron llegar a esfuerzos de potencia aeróbica máxima y potencia anaeróbica submáxima y hasta casi máxima, lo que pudo haber llevado a mayor concentración de lactato a partir de su buena condición atlética.

Tanto en las mujeres como en los hombres la concentración de lactato en el rango superior fue de $15 \mathrm{mmol} / \mathrm{l}$, relacionado, en este caso, con los deportistas que mejor $\mathrm{VO}_{2 \max }$ tienen, lo que lleva a pensar en la importante correlación que existe entre una buena condición física y la cantidad de ácido láctico que se puede acumular en el esfuerzo para poderlo soportar. Sin embargo, no es posible generalizar este comportamiento del lactato, puesto que hubo deportistas cuyo desempeño físico no fue el ideal y, no obstante, llegaron a concentrar niveles altos. Esta situación podría explicarse desde el ámbito deportivo, si se toma en cuenta el hecho de que el lactato puede ser reutilizado para generar energía durante el ejercicio y también durante la recuperación. Según Giraldo y Sánchez (1998), el músculo produce y consume lactato y al aumentar sus niveles en la sangre los músculos inactivos, y también otros tejidos, pueden 
capturar y utilizar este ion. Además, se debe reconocer el hecho de que a través del entrenamiento se pueden modificar los mecanismos de aclaramiento del lactato, lo que conduce a ser un deportista con buena utilización y tolerancia al mismo. De ahí que se deba entrenar en la zona de tolerancia al lactato, más aún si hablamos de deportistas obligados por su deporte a trabajar, ya sea por momentos o de forma continua, en su presencia.

Con respecto a la prueba, los deportistas, en la medida en que aumentan las etapas, aumentan también la velocidad de desplazamiento (aumento progresivo), lo que seńaló velocidades que estuvieron, para el caso de este grupo, entre 12 y $14 \mathrm{~km} / \mathrm{h}$, es decir, etapas 10 y 11 . En ese momento de la prueba, el deportista ha demostrado buena capacidad aeróbica-anaeróbica, puesto que sus desplazamientos son más rápidos (4 y 5 segundos, en promedio, para recorrer 20 metros) y, por lo tanto, se encuentran haciendo esfuerzos ubicados en el umbral, esto lo hace llegar, por obvias razones, a esa zona donde la acumulación del lactato es superior a la capacidad que tiene el deportista de poder eliminarlo efectivamente, hasta llegar al punto de no ser capaz de contrarrestar el esfuerzo solicitado y, a la postre, a la finalización de la prueba.

De acuerdo con lo planteado por Sergeyevich y Dmitriyevich (2001),

la concentración es tan alta (lactato en sangre mayor de $15-20 \mathrm{mmol} / \mathrm{l}$ ) que las fibras musculares, especialmente las mitocondrias, no pueden funcionar en forma normal. En un trabajo de este tipo el rendimiento de los músculos se determina por la capacidad para superar o no en el tiempo los cambios de la acidosis. (p. 300)

En consonancia con lo anterior, los deportistas que son capaces de llegar en la prueba a una etapa elevada demuestran con ello, cada vez más, una mayor capacidad de resistencia que no solo se evidencia en el nivel de $\mathrm{VO}_{2 \max }$, sino también en la asimilación con respecto al esfuerzo que se ha alcanzado gracias al entrenamiento y a los cambios que este genera en sus mecanismos fisiológicos para trabajar eficazmente. En el hígado, el lactato se puede convertir en glucosa, esta glucosa puede retornar el músculo y ser utilizada por este como fuente de energía que prolonga la glucólisis muscular (Ciclo de Cori), el lactato funciona entonces como un intermediario metabólico importante que puede rápidamente intercambiarse entre compartimentos musculares y entre diferentes tejidos. 
En términos generales, el promedio de la concentración de lactato en la tercera muestra fue en mujeres de 6,8 y en hombres de y $9,23 \mathrm{mmol} / \mathrm{l}$, lo que significó una disminución de acuerdo con lo concentrado en la muestra 2 , donde se evidenció el umbral. Estos niveles disminuyeron paulatinamente en la medida en que pasaron las horas y la recuperación se estableció más allá de los diez minutos determinados para la última muestra. De la misma forma, se encontró una recuperación satisfactoria de la frecuencia cardiaca promedio. El comportamiento tanto de la concentración del lactato como de la frecuencia cardiaca, a nivel general en los tres momentos, evidencia que a nivel de la concentración de lactato hubo un incremento del $640 \%$ postest con respecto a la muestra basal, y en la tercera muestra disminuyó $23,8 \%$ con respecto a la segunda. Mientras que la frecuencia cardiaca en el postest se incrementó en un $103 \%$ con respecto a las condiciones basales y posteriormente disminuyó en un $38 \%$ tras 10 minutos de reposo.

\section{Conclusiones}

El promedio general de la frecuencia cardiaca de los voleibolistas a nivel basal, posesfuerzo y en recuperación fue de 95, 193 y 119 ppm respectivamente. Lo que mostró que a nivel general el grupo presenta una adecuada recuperación.

Con respecto al $\mathrm{VO}_{2 \max }$ de la muestra evaluada, se observó un valor de 52,2 $\mathrm{ml} / \mathrm{kg} / \mathrm{min}$ en promedio, sin embargo, se presentó mayor consumo por parte de los hombres $(55,1 \mathrm{ml} / \mathrm{kg} / \mathrm{min})$ con respecto a las mujeres $(48,2 \mathrm{ml} / \mathrm{kg} / \mathrm{min})$.

Con relación a la concentración de lactato en función del momento de la toma de la muestra, los datos corroboran que existen diferencias entre las medias de la concentración de lactato entre la muestra basal, posesfuerzo y tras 10 minutos de reposo $(\mathrm{p}<0.05)$, a nivel general y según el sexo. Sin embargo, no hubo diferencias estadísticamente significativas al comparar cada uno de los momentos $($ basal $\mathrm{P}$-valor $=0,9542$, postest $\mathrm{P}$-valor $=0,3677$, reposo $\mathrm{P}$-valor $=$ 0,0973) entre hombres y mujeres.

De acuerdo con el promedio de la concentración de lactato, a nivel basal, los deportistas se encontraban en la zona aeróbica compensadora. 
Con respecto a la segunda muestra se observó que el $88 \%$ de los voleibolistas evaluados debido a su concentración de lactato, se encontraban en la zona anaeróbica estimulante.

En términos generales, el promedio de la concentración de lactato en la tercera muestra, es decir, tras diez minutos de reposo, presentó una disminución general del 23, 8\%.

\section{Referencias}

Ahonen, J., Lahtinen, T., Sandström, M., Pogliani, G., \& Wirhed, R. (2001). Kinesiología y anatomía aplicada a la actividad física. Paidotribo.

Alonso-Curiel, D., Campo-Vecino, J., Balsalobre, C., Tejero, C., \& Ramírez-Parenteau, C. (2012). Respuesta láctica de atletas de élite ante un entrenamiento específico para la prueba. Apunts. Educación Física y Deportes, 1(107), 90-96. https://www.raco.cat/index. $\mathrm{php} /$ ApuntsEFD/article/view/252858

American College of Sport Medicine (2006). Resource Manual for Guidelines for Exercise and Prescription (5a. ed.). Lippincott Williams \& Wilkins.

Astrand, P., \& Rodahl, K. (1986). Textbook of work physiology: Physiological bases of exercise (3ra ed.). McGraw-Hill.

Atkins, P. (2007). Las moléculas de Atkins. Ediciones Akal.

Balsalobre, C., Tejero, C., Campo, J., \& Alonso, D. (2014). la exposición hipóxica como medio para aumentar el rendimiento deportivo: ¿mito o realidad? Revista internacional de medicina y ciências de la actividad fisica y del deporte, 14(53), 183-98. https://www.redalyc.org/ articulo.oa?id=54230508012

Cabral, B., Cabral, S., Toledo, I., Silva, P., Ferreira, H., \& Knakfuss, M. (2011). Antropometria E Somatotipo: Factores determinantes na seleção de atletas no voleibol Brasileiro. Revista Brasileira de Ciências do Esporte, 33(3), 733-746. http://revista.cbce.org.br/index.php/ RBCE/article/view/1012/688

Earle, R., \& Baechle, T. (2007). Principios del entrenamiento de la fuerza y del acondicionamiento físico (2a. ed.). Editorial Médica Panamericana.

Engel, F., Härtel, S., Wagner, M., Strahler, J., Bös, K., \& Sperlich, B. (2014). Hormonal metabolic and cardiorespiratory responses of young and adult athletes to a single session of high-intensity cycle exercise. Pediatric Exercise Science, 26(4), 485-494.

García-Cardona DM, Sánchez-Muñoz OE, Cabrera-Arismendy CE, Restrepo-Cortés B. (2017). Perfil lipídico, antropométrico y condición física de estudiantes deportistas universitarios. Rev Univ. Salud, 19(2), 267-279.

Giraldo, J., \& Sánchez, M. (1998). El lactato como posible factor del mecanismo de fatiga muscular. Colombia Médica, 29(2-3), 87-91. http://colombiamedica.univalle.edu.co/ index.php/comedica/article/view/95/94 
Ivoilov, A. (1986). Voleibol: técnica, táctica y entrenamiento. Stadium.

López, A. (2006). Medicina del deporte: Control biomédico del entrenamiento deportivo y control morfo-funcional para diferentes niveles de preparación fisica. Universidad Libre.

López, A. (2009). Control biomédico del entrenamiento en diferentes deportes y en deportistas de empresas (1 $1^{\text {a }}$ ed.). Universidad Libre.

López, P. (2002). Mitos y falsas creencias en la práctica deportiva. Barcelona: INDE.

Norton, K., \& Olds, T. (1996). Antropométrica. Southwood Press.

OMS, Organización Mundial de la Salud. (2016). Obesidad y sobrepeso. Nota descriptiva 311(1).

Pérez, Y., Portela, Y., Cantero, M., \& Rodríguez, E. (2014). Características antropométricas de jugadores de voleibol en la Universidad de las Ciencias Informáticas. EmásF: Revista Digital de Educación Física, 5(30), 8-11.

Ramos, S., Melo, L., \& Alzate, D. (2007). Evaluación antropométrica y motriz condicional de los escolares de 7 a 18 años de edad. Universidad de Caldas.

Sergeyevich, V., \& Dmitriyevich, V. (2001). Fisiología del deportista (2ª ed.). Paidotribo.

Urdampilleta, A., Martínez-Sanz, J., \& López-Grueso, R. (2013). Valoración bioquímica del entrenamiento: herramienta para el dietista-nutricionista deportivo. Revista Española de Nutrición Humana y Dietética, 17(2), 73-83. http://renhyd.org/index.php/renhyd/article/ view/14

Zanella, A., Mazini, M., Gama, D., De Oliveira, G., Costa, O., \& Paula, S. (2015). Consumo máximo de oxigênio de jogadores de voleibol: comparação entre posiçôes de jogo. Revista Brasileira de Prescrição e Fisiologia do Exercício, 9(54), 404-409. http://www.rbpfex.com. br/index.php/rbpfex/article/view/846/719 